\title{
Molecular characterization of two glutathione peroxidase genes of Panax ginseng and their expression analysis against environmental stresses
}

\author{
Yu-Jin Kim ${ }^{1}$, Moon-Gi Jang ${ }^{1}$, Hae-Yong Noh, Hye-Jin Lee, Johan Sukweenadhi, Jong-Hak Kim, Se-Yeong Kim, \\ Woo-Saeng Kwon, Deok-Chun Yang *
}

Department of Oriental Medicinal Materials and Processing, College of Life Science, Kyung Hee University, Suwon 449-701, Republic of Korea

\section{A R T I C L E I N F O}

Article history:

Accepted 30 October 2013

Available online 22 November 2013

\section{Keywords:}

Abiotic stress

Glutathione peroxidase

Gene expression

Panax ginseng

\begin{abstract}
A B S T R A C T
Glutathione peroxidases (GPXs) are a group of enzymes that protect cells against oxidative damage generated by reactive oxygen species (ROS). GPX catalyzes the reduction of hydrogen peroxide $\left(\mathrm{H}_{2} \mathrm{O}_{2}\right)$ or organic hydroperoxides to water or alcohols by reduced glutathione. The presence of GPXs in plants has been reported by several groups, but the roles of individual members of this family in a single plant species have not been studied. Two GPX cDNAs were isolated and characterized from the embryogenic callus of Panax ginseng. The two cDNAs had an open reading frame (ORF) of 723 and 681 bp with a deduced amino acid sequence of 240 and 226 residues, respectively. The calculated molecular mass of the matured proteins are approximately $26.4 \mathrm{kDa}$ or $25.7 \mathrm{kDa}$ with a predicated isoelectric point of 9.16 or 6.11, respectively. The two PgGPXs were elevated strongly by salt stress and chilling stress in a ginseng seedling. In addition, the two PgGPXs showed different responses against biotic stress. The positive responses of PgGPX to the environmental stimuli suggested that ginseng GPX may help to protect against environmental stresses.
\end{abstract}

(c) 2013 Elsevier B.V. All rights reserved.

\section{Introduction}

In living organisms, reactive oxygen species (ROS) are generated during metabolic processes, such as photosynthesis and respiration (Rodriguez Milla et al., 2003). In addition, plants are easily exposed to biotic and abiotic stresses, which can trigger a dramatic ROS increase in the intracellular environment, resulting in oxidative stress (Grene, 2002). Some ROS, such as superoxide radicals, hydroxyl radicals, and hydrogen peroxide $\left(\mathrm{H}_{2} \mathrm{O}_{2}\right)$, are potentially toxic to cells and can damage biological molecules when produced at high rates (Halliwell and Gutteridge, 2007). To maintain low cellular concentrations of ROS, plants have developed antioxidant enzymes and metabolites at variable amounts in different tissues, cells, and organelles.

Glutathione peroxidase (GPX, EC 1.11.1.9) is the principal cellular enzyme capable of protecting organisms from oxidative damage by catalyzing the reduction of lipid peroxides and other organic peroxides to the corresponding alcohols using thioredoxins as the preferred electron donors (Mari et al., 2009; Tanaka et al., 2005). GPXs have been extensively investigated in animals, and they are classified into six groups

Abbreviations: GPX, glutathione peroxidase; ROS, reactive oxygen species; $\mathrm{H}_{2} \mathrm{O}_{2}$, hy drogen peroxide; CS, Citrus sinensis; AF, Avena fatua; At, Arabidopsis thaliana; Bc, Brassica campestris; So, Spinacia oleracea; Ha, Helianthus annuus; Le, Lycopersicon esculentum; Ps, Pisum sativum; Hv, Hordeum vulgare; Or, Oryza sativa; Cc, Chinese cabbage; Lj, Lotus japonicas.

* Corresponding author. Tel.: +82 31201 2100; fax: +82 312022687.

E-mail address: dcyang@khu.ac.kr (D.-C. Yang).

1 These authors contributed equally to this work that differ in amino acid sequence, structure, substrate specificity, and subcellular localization (Herbette et al., 2007; Ursini et al., 1995). Although much less is known about plant GPXs, they have been shown to be similar in terms of amino acid sequences to the mammalian GPX4 enzyme type, which comprises the selenium-dependent phospholipid hydroperoxide GPX (PHGPX) (Herbette et al., 2007).

Several GPX cDNA clones have been isolated from various plants including Citrus sinensis (Holland et al., 1993), Avena fatua (Johnson et al., 1995), Arabidopsis thaliana (Sugimoto and Sakamoto, 1997), Brassica campestris (Eshdat et al., 1997), Spinacia oleracea (Sugimoto et al., 1997), Helianthus annuus (Roeckel-Drevet et al., 1998), Lycopersicon esculentum (Depege et al., 1998), Pisum sativum (Mullineaux et al., 1998) Oryza sativa (Li et al., 2000), and Lotus japonicas (Ramos et al., 2008). To date, few studies have been published describing the family of plant GPXs. The plant GPX family may comprise up to six members that are distributed in different subcellular compartments (Navrot et al., 2006). AtGPX1-AtGPX8 have been identified in A. thaliana, and their expressions are differentially induced in response to various abiotic stresses and plant hormones (Navrot et al., 2006; Rodriguez Milla et al., 2003). The major function of GPXs in plants appears to be the scavenging of phospholipid hydroperoxides and thereby the protection of cell membranes from peroxidative damage (Gueta-Dahan et al., 1997). Recent data showed that some GPXs may also be involved in redox transduction under stressful conditions (Miao et al., 2006).

The present study examined the phylogenetic relationship of ginseng GPX and that of other organisms, and to contribute to the understanding of the possible role of ginseng GPX. Despite their importance 